\title{
Correction to: Comparative Proteomic Analysis Reveals Metformin Improves the Expression of Biomarkers of Endometrial Receptivity in Infertile Women with Minimal/Mild Endometriosis
} Xin Huang ${ }^{1,2,3} \cdot$ Li Xiao $^{1,2} \cdot$ Ying Long $^{1,2} \cdot$ Tianjiao Pei $^{1,2} \cdot$ Bin Luo $^{1,2} \cdot$ Tianji Liao $^{1,2} \cdot$ Yujing Li $^{1,2,3} \cdot$ Huili Zhu $^{1,2}$
Yunwei Ouyang

Published online: 3 February 2022

(c) Society for Reproductive Investigation 2022

Correction to: Reproductive Sciences

https://doi.org/10.1007/s43032-022-00869-3

This article was updated to include the article note:

Xin Huang, Li Xiao and Ying Long have contributed equally to this work and share first authorship.

The original article can be found online at https://doi.org/10.1007/ s43032-022-00869-3.

Wei Huang

weihuang64@163.com

1 Division of Reproductive Medicine, West China Second University Hospital of Sichuan University, Chengdu 610041,

Sichuan, China

2 Key Laboratory of Birth Defects and Related Diseases of Women and Children of Ministry of Education, Chengdu 610041, Sichuan, China

3 NHC Key Laboratory of Chronobiology, Sichuan University, Chengdu 610041, Sichuan, China 